MIDPI sciforum
MOL2NET, International Conference Series on Multidisciplinary Sciences http://sciforum.net/conference/mol2net-03

\title{
Luciferase as a research technique for biological processes
}

\section{Paramo Ramirez Arely (arelyparamo@ hotmail.com) a,", Gonzalez Luna Alma Delia} (almadelia097@gmail.com) ${ }^{\mathrm{b}}$, Galeana Rojas Maria Fernanda ${ }^{\mathrm{c}}$,Jauregui Perez Laura Jannete (laurajannete@gmail.com) d

${ }^{a}$ Division of Natural and Exact Sciences, Universidad de Guanajuato, Guanajuato, Guanajuato Mexico.

${ }^{b}$ Campus Regional Mixteca sede Izúcar de Matamoros, Faculty of Biological Sciences Benemérita Universidad Autónoma de Puebla, Puebla.

c Department Technologies Information, Universidad De La Salle Bajío, León, Guanajuato, Mexico.

${ }^{a}$ Division of Natural and Exact Sciences, Universidad de Guanajuato, Guanajuato, Guanajuato Mexico.

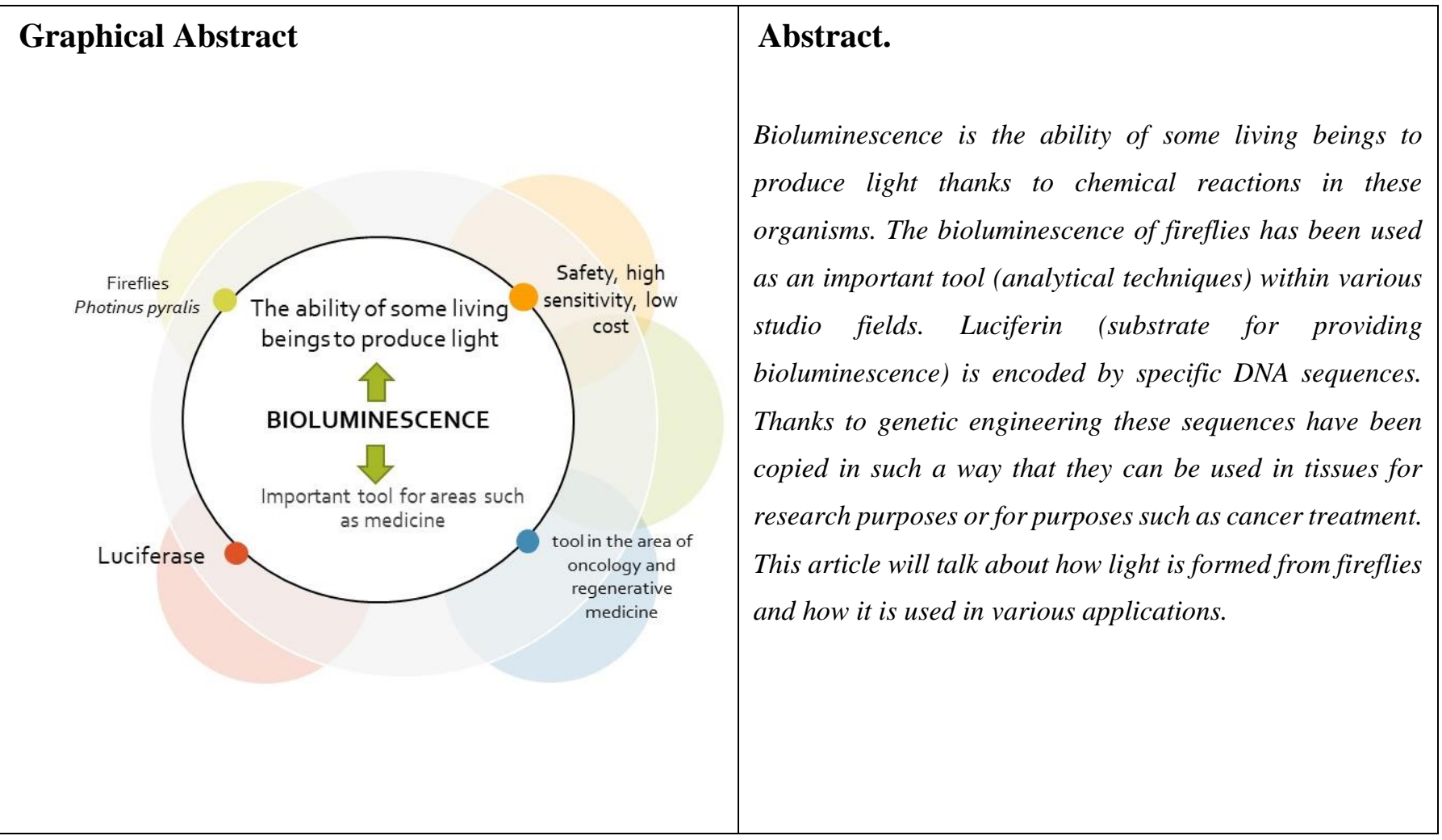

\section{Introduction}

The bioluminescence of fireflies has been used as an important tool (analytical techniques) within various fields of study. The emission of yellow green light from fireflies is generated by the catalytic conversion of chemical energy enzymes using luciferin in light as a substrate. The enzyme is called luciferase and the substrate luciferin. As mentioned, firefly luciferin and luciferase is used as an analytical study tool. The mostly studied luciferase enzyme is from the organism Photinus pyralis of North America.

The reaction carried out for bioluminescence to occur requires adnosin-5'-triphosphate (ATP) as a cofactor. ATP is the universal energy of living organisms, ATP luciferin-luciferase detection trials have 
become standard methods for detecting microbes in food and in the pharmaceutical industry as clinical settings; In addition to this, luciferin-luciferase has been a convenient tool for the distinction of living cells and dead cells.

One advantage of bioluminescence compared to fluorescence is that the former does not require an excitation light source, the images are generated with low background signals.

The reaction mechanism for bioluminescence production requires ATP, $\mathrm{Mg}(2+)$ and molecular oxygen, taking within the entire reaction oxidative decarboxylation of luciferin, acetylation, finally a photon of visible light is released, which is visible as the bioluminescence coming from the firefly.

For the analysis of biological processes, colors other than the bioluminescent yellow-green color are necessary for both in vivo and in vitro assays. Thus, the use of luciferase from other species and/or mutations of the enzyme constitutes strategies for the emission of light with different colors from the reaction of luciferin-luciferase. Tripluc® is a commercial tricolor luciferase consisting of a system developed by mutant luciferasas, said system consists of green (550 nm), orange (580) and red (630). The marketed luciferase genes can be classified into two types. The first type are derived from marine organisms, these structures are categorized as imidazopiradinones; the second type include firefly luciferase genes. The last type are commonly chosen for in vivo testing by bioluminescent imaging (BLI) giving a wavelength emission in the range of $460-630 \mathrm{~nm}$, a strategy to modulate the wavelength emission is to alternate substrates in the structure.

Thus modifying the structure to obtain a high wavelength range of the lucifersa-catalytic is an advantage since this is possibly an important factor that determines the maximum wavelength. Ideally the variability that can be obtained with the modification of the structure can be applied as immobilized marks for specific molecules for multiple tests using luciferase, emitting different wavelengths.

The application of in vivo images resides in an important tool in the area of oncology and regenerative medicine. The perfect imaging modality should have the following properties: Safety, high sensitivity, without genetic modifications, low cost, etc., it is worth mentioning that not all techniques used in the area of medicine have all the properties these include: X-ray CT, positron emission tomography, MRI. Images by BLI are categorized as optical imaging techniques and these have low-cost, short acquisition and high sensitivity properties for molecular and cellular images.

\section{Conclusions}

As mentioned above, luciferin is encoded by specific DNA sequences; Thanks to genetic engineering these sequences have been copied in such a way that they can be used in tissues for research purposes or for purposes such as cancer treatment. The introduction of this protein into hundreds of tissues for expression has helped to perform in vivo processes to understand biological processes.

Biotechnology has required proteins such as luciferase to solve specific problems in human tissues, the usefulness of this protein in the area of medicine is extremely beneficial, the study of proteins involved in avoiding diseases such as Alzheimer's, Parkinson's, or some cancers.

\section{References}

Faridy Panohaya García Dr. Arturo Olivares Pèrez Q.I. Israel Fuentes Tapia . (diciembre 2004). Conceptos y bibliografía sobre la fotoluminiscencia y procesos similares. Mexico : INAOE. 
H.Gonzalez-Díaz et,al. (2016). Multicolor bioluminiscence Obtained Using Firefly Luciferin. current topics in medicinal Chemistry, 16, 2648-2655.

Viviani,V.R.. The origin, diversity, and structure function relationships of insects luciferases. Cell. Mol. Life Sci.,2002, 59(11),1833-1850.

McElroy,W. D.; The energy source for bioluminescence in a isolated system. Proc. Natl.Acad.Sci. USA, 1947,33 (11); 342-345 